\title{
Optimal Power Control in Wireless Data Networks with Outage-based Utility Guarantees
}

\author{
Subhrakanti Dey and Jamie Evans \\ Department of Electrical and Electronic Engineering \\ University of Melbourne \\ Parkville, Victoria 3010, Australia \\ $\{$ s.dey, j.evans\}@ee.mu.oz.au
}

\begin{abstract}
In this paper, we address the problem of optimal power control in the uplink of a single cell of a multiuser interference limited wireless data network with Rayleigh fading. We propose a frame outage-based utility function that is optimized for each user. We consider two optimization problems: (1) Maximizing the minimum utility and (2) minimizing the total transmitted power subject to each user meeting their minimum utility targets. We propose simple iterative algorithms to solve these optimization problems which are seen to converge rapidly to the optimal solutions. Some numerical studies are carried out to illustrate the performance of these algorithms, demonstrating that one can achieve large utility values by spending a fairly small amount of transmit powers provided one can employ an appropriate multiuser detector at the receiver to guarantee a substantial processing gain. This allows data communication to take place with a low probability of frame outage even over rapidly fading Rayleigh channels. The power control algorithm needs to update at a much slower rate than the rate of fast channel fading, thus making it easy to implement in practice.
\end{abstract}

\section{INTRODUCTION}

Efficient power control is an important problem in wireless networks for reducing radiation levels and extending battery lives of mobile devices. Furthermore, it is crucial for interference limited networks such as CDMA where optimal control of transmit powers can lead to effective interference minimization and to an increase of capacity in the network. Power control in the uplink of a wireless network has been studied as a constrained optimization problem in [1] [2] [3] and many others (see references in these papers as well). The basic objective of this problem has been to minimize total transmitted powers of mobile users subject to each user meeting their Quality of Service (QoS) guarantees. Centralized and distributed algorithms have been found to update the powers of the mobiles whenever the channel characteristics change, assuming that the rate of change of the channel is fairly slow, allowing the change to be tracked accurately. The problem has also been considered in a singlecell [2] as well as a multi-cell framework [3]. Convergence of these algorithms has been proved for both synchronous and asynchronous CDMA applications. In the case where channel link gains are not exactly known but are estimated only, [4] has proposed a distributed power control algorithm based on interference measurements at the receiver which is proved to converge in the mean-square sense using stochastic approximation based ideas.

However, most of these earlier works address the power control problem with the assumption that the wireless link gains are time-invariant or slowly time-yarying. This allows the bit-error-rate (BER) or the signal-to-interference ratio (SIR) for a particular user to be used as the appropriate QoS when defining the constraints in the optimization problem. In the case of voice communications over wireless channels, it is well known that rapidly fading wireless channels make such constraints on a minimum achievable SIR difficult to achieve. This is true because tracking fast variations in the channel may become difficult, or updating the power efficiently at such a rapid rate may also pose inconvenience. Therefore one needs to define the so-called "outage probability" to characterize the probability that the SIR falls below that minimum acceptable threshold. The constraints in the optimization problem are then replaced by maximum allowable outages. A recent work that treats such an optimization problem for Rayleigh fading channels is [5], where convex optimization techniques are used to solve various optimal power control problems with outage probability specifications. In [6], an optimal power control problem with outage probability specifications is solved using a simple iterative technique with guaranteed convergence for the Rayleigh fading case. The authors also showed that one can achieve fairly small power utilization if one combines an appropriate multiuser detector (such as a linear minimum mean square (LMMSE) detector) with the power control algorithm.

While there has been a huge amount of work on power control for voice communications over wireless, new power control techniques for data communication over wireless are still emerging. While data rates can be adapted for achieving better quality of service even in rapidly fading channel conditions, power control remains an important problem in the context of fixed-rate data communications over wireless (see [7]. A recent paper [8] (see also references therein) shows how game theoretic ideas can be used to include pricing in solving the power control problem for data over wireless. This paper considers a single-cell CDMA network where each user wants to optimize their own utility functions, thus setting up a non-cooperative multi-person game problem. By including pricing, the authors show that one can come up with a distributed solution to the problem that is Pareto optimal. A centralized version of the problem is also studied in [9]. Similar game theoretic ideas are also explored for power control with capacity optimization in [10]. These works however, assume that the channel is time-invariant or slowly time-varying and thus can be estimated fairly 
accurately. While there have been ad-hoc power and rate control algorithms for fading channels [11], there has been no systematic study for power control over data networks with rapidly fading channels using outage (for frame error rates rising above a certain acceptable value) based constraints.

In our work, therefore, we propose an outage-based utility function for each user under Rayleigh fading conditions. This utility function represents the ratio of frame success probability per unit of power spent. We consider the uplink of a single-cell interference limited network (such as CDMA) with a generic multiuser detector at the receiver that provides an appropriate processing gain. We consider the problem as a centralized optimization problem where the power control algorithm is really implemented at the base station, and the power updates are communicated to the mobile with a delay less than the time between consecutive power updates. We consider two optimization problems: (1) maximizing minimum utility and (2) minimizing sum power subject to each user meeting a minimum acceptable utility value. The algorithms developed are simple iterative procedures which are seen to converge within a few iterations. We also study the performance of these algorithms numerically, illustrating the fact that an appropriate multiuser detector can be employed at the receiver to achieve large utility values even with small power utilization and an acceptably high frame success rate.

\section{SySTEM MOdEL AND OUTAGE-BASED UTILITY}

We consider a single cell in a cellular wireless communication system with $K$ users simultaneously transmitting $L$ information bits in packets (frames) of $M(M>L)$ bits at a fixed rate of $R$ bits/second. In this work, we assume that the transmission rate for all users is fixed at $R$. Denote the $i$ th user's transmission power as $p_{i}, i=1, \ldots, K$. For a time invariant wireless channel, an appropriate utility function that the $i$-th user chooses to optimize was given in [8] as

$$
u_{i}^{f}=\frac{\operatorname{LRf}\left(\gamma_{i}\right)}{M p_{i}}
$$

where $\gamma_{i}$ is the signal-to-interference ratio (SIR) for the $i$ th user and $f($.$) is an efficiency function that approximates$ the frame success rate (FSR) (which is equivalent to the probability of all bits in a frame being received correctly).

\section{Frame Outage-based Utility}

In the case of a randomly time varying channel, the SIR itself is a random process and thus we need to find an alternative definition for a utility function which is based on a frame outage probability (FOP). The FOP for the $i$-th user is defined as the probability that the FSR for the $i$-th user falls below a minimum acceptable FSR, $F S R_{\text {min }}$. Denoting the FOP for the $i$-th user as $O_{i}^{F}$, we can express the utility function for the $i$-th user as

$$
u_{i}=\frac{L R\left(1-O_{i}^{F}\right)}{M p_{i}}
$$

Assuming BPSK modulation for data transmission and that SIR is constant over a frame (that is the channel fading is static over a frame), we can compute the FSR for the $i$-th user as

$$
F S R_{i}=\left[1-Q\left(\sqrt{2 S I R_{i}}\right)\right]^{M}
$$

where $Q(x)=\frac{1}{\sqrt{2 \pi}} \int_{x}^{\infty} \exp \left(-t^{2} / 2\right) d t$. Since $Q(x)$ is a monotonically decreasing function of $x$, the FOP can now be simply related to the traditional concept of outage probability used in voice communications as $P\left(S I R_{i}<\right.$ $S I R_{\text {min }}$ ) where given a $F S R_{\min }$ one can easily compute the corresponding $S I R_{\min }$.

Note also that when $p_{i}=0, O_{i}^{F}=1$. However, we assume that $\lim _{p_{i} \rightarrow 0} \frac{\left(1-O_{i}^{F}\right)}{p_{i}}$ exists and is finite. Later on, for a specific channel fading statistic, we shall show that indeed, this assumption holds true.

\section{Derivation of Outage-based Utility}

In order to derive the expression for the outage-based utility $u_{i}$ as defined above, we need to specify the statistical model of the wireless channel gains for each user transmitting data. We also assume here that there is an appropriate multiuser detector employed at the receiver that provides a processing gain of $P_{G}$. In order to keep discussions simple, we settle on a generic multiuser detector, but note that this work can be easily extended to consider specific forms of multiuser detectors such as linear minimum mean square error multiuser detector (LMMSE MUD) and the optimization can be seen as a joint optimization over the users' transmitter powers and the multiuser receiver filters (as in [6]).

Denote the channel gain from user $j$ 's transmitter to the $i$-th user's receiver as $H_{i j}=G_{i j} F_{i j}$ where $G_{i j}$ is taken to be time-invariant and $F_{i j}$ is a random process denoting the rapidly time-varying component of the channel. In reality, $G_{i j}$ may be slowly time-varying (e.g, distributed according to a log-normal shadowing model), but its slow variation allows the receiver to track it accurately using sophisticated channel estimation algorithms. For this reason, we will assume in this work that $G_{i j} \forall i, j$ are time-invariant, that is they represent the time-invariant channel attenuation due to the distance between the transmitter and the receiver. We also assume that $G_{i j}$ implicitly includes the processing gain not just due to the spreading factor, but also the one achieved through the use of the multiuser detectors at the receivers. With this in mind $G_{i i}$ is expected to be much larger than $G_{i j}, j \neq i, i, j=1, \ldots, K$.

For the purpose of this paper, we assume that $F_{i j}, \forall i, j$ are statistically independent Rayleigh-distributed random variables with unit mean. This is traditionally known as the Rayleigh/Rayleigh fading environment which is applicable in dense urban areas where none of the users has any direct line of sight. Note that the instantaneous SIR for the $i$-th user 
is given as

$$
S I R_{i}=\frac{G_{i i} F_{i i} p_{i}}{\sum_{k \neq i} G_{i k} F_{i k} p_{k}+\sigma^{2}}
$$

where the term in the numerator denotes the received power of the $i$-th user, whereas the denominator denotes the total interference due to all other users' received power at the $i$-th receiver and the noise variance $\left(\sigma^{2}\right)$ due to the white channel noise and thermal noise at the receiver.

For simplicity, we assume that the $F S R_{\min }$ for all users is the same although this work can easily be extended to multiclass users with different quality of service (QoS) guarantees. The corresponding $S I R_{\min }$ then is denoted by $\gamma_{t h}$ and is computed as

$$
\gamma_{t h}=\left[\operatorname{erfcinv}\left(2\left(1-F S R_{\text {min }}^{1 / M}\right)\right)\right]^{2}
$$

where erfcinv denotes the inverse complementary error function erfc which is related to the $Q$ function as $Q(x) \triangleq$ $0.5 \operatorname{erfc}\left(\frac{x}{\sqrt{2}}\right)$.

Once $\gamma_{t h}$ is determined, the FOP for the $i$-th user is calculated as

$$
\begin{aligned}
O_{i}^{F} & =P\left(S I R_{i}<\gamma_{t h}\right) \\
& =1-\exp \left(-\frac{\sigma^{2} \gamma_{t h}}{G_{i i} p_{i}}\right) \prod_{k \neq i} \frac{1}{1+\frac{\gamma_{t h} G_{i k} p_{k}}{G_{i i} p_{i}}}
\end{aligned}
$$

For a derivation of this outage probability and outage probabilities for other fading distributions, see [12] and [13] and references therein.

Substituting this in (2), we obtain the expression for our utility function as

$$
u_{i}=\frac{L R}{M p_{i}} \exp \left(-\frac{\sigma^{2} \gamma_{t h}}{G_{i i} p_{i}}\right) \prod_{k \neq i} \frac{1}{1+\frac{\gamma_{t h} G_{i k} p_{k}}{G_{i i} p_{i}}}
$$

It can be easily verified that in this case, $\lim _{p_{i} \rightarrow 0} \frac{1-O_{i}^{F}}{p_{i}}=0$.

\section{OPtIMAL POWER CONTROL}

In this section, we consider two optimization problems: (1) Maximize the minimum utility while each individual user's power is constrained between zero and maximum value and (2) Minimize sum of all users' transmitted powers such that each user meets a minimum outage-based utility guarantee. These two problems are studied in the next two subsections.

\section{A. Maximizing Minimum Utility}

In this subsection, we consider the problem:

\section{Problem A:}

Maximize $\min _{i} u_{i}$ over $\mathbf{p}=\left(\begin{array}{llll}p_{1} & p_{2} \ldots p_{K}\end{array}\right)^{\prime}$ such that $0<$ $p_{i} \leq p_{\max }, \forall i$.

In order to study this problem, let us first look at the nature of the utility function as a function of a user's own power when other users' powers are kept fixed. Figure 1 shows a typical utility plot (for a system with 4 users) for user 1 when

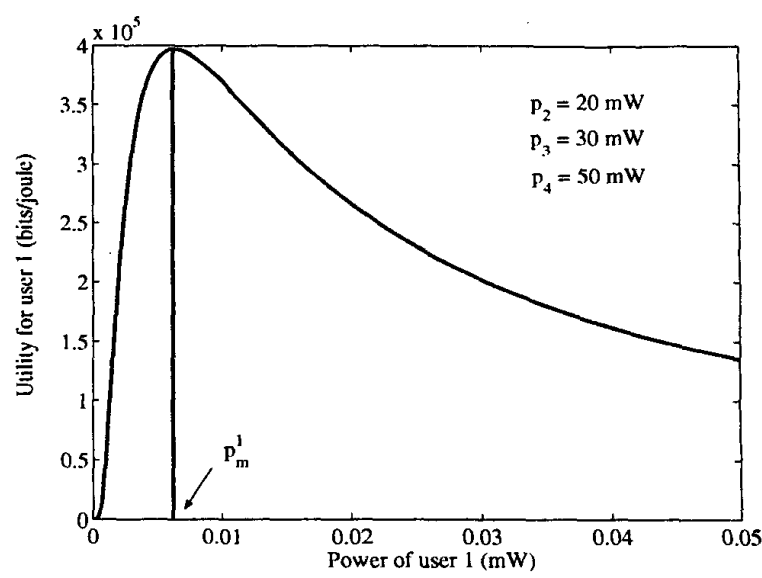

Fig. 1. Plot of Utility of User 1 versus $p_{1}$

the powers for the three remaining users in the system are kept fixed.

Notice that $u_{1}$ goes to zero as $p_{1} \rightarrow 0$ or $p_{1} \rightarrow \infty$. There is a unique maximum at the value $p_{m}^{1}$. The fact that there is a unique maximum can be proved carrying out the usual analysis on $\frac{\partial \ln u_{1}}{\partial p_{1}}$ and showing that it has a unique maximum. The algebra is straightforward and excluded from this paper. This also implies that $u_{i}$ is a strictly monotonically increasing function of $p_{i}$ for $0<p_{i} \leq p_{m}^{i}$.

Now suppose that there is a solution to Problem A given by $\mathbf{p}^{*}=\left(p_{1}^{*} p_{2}^{*} \ldots p_{K}^{*}\right)^{\prime}$. The following Lemma states that $0<p_{i}^{*} \leq p_{m}^{i}$.

Lemma 3.1: If $\mathbf{p}^{*}=\left(\begin{array}{lll}p_{1}^{*} & p_{2}^{*} & \ldots \\ p_{K}^{*}\end{array}\right)^{\prime}$ is a solution to Problem A, then $0<p_{i}^{*} \leq p_{m}^{i}, \forall i$.

Proof: Note that if $\mathbf{p}^{*}=\left(p_{1}^{*} p_{2}^{*} \ldots p_{K}^{*}\right)^{\prime}$ is a solution to Problem A, then $\mathbf{p}^{*}$ maximizes the minimum utility. Suppose $p_{i}>p_{m}^{i}$ for some $i$. In that case, notice that just decreasing $p_{i}$ would increase the utility of user $i$ as well as the utilities of other users, as $u_{i}$ can be seen (from (7)) to be a strictly monotonically increasing function of $p_{k}, k \neq i$ as $p_{k}$ decreases. Clearly, this implies that $\mathbf{p}^{*}$ does not maximize the minimum utility. Therefore $p_{i}^{*} \leq p_{m}^{i}$. Since $i$ is arbitrary, the proof is complete.

Lemma 3.1 leads to the following additional Lemma.

Lemma 3.2: Suppose $\mathbf{p}^{*}$ maximizes the minimum utility. If $u_{i}$ evaluated at $\mathbf{p}^{*}$ is denoted as $u_{i}^{*}$, then all $u_{i}^{*}, i=$ $1,2, \ldots, K$ are equal.

Proof: Recall that for $0<p_{i} \leq p_{m}^{i}, u_{i}$ is a strictly monotonically increasing function of $p_{i}$ and a strictly monotonically decreasing function of $p_{k}, k \neq i$ as $p_{k}, k \neq i$ increases. Now suppose that not all $u_{i}^{*}$ are equal. Choose an index $j$, such that $u_{j}^{*}>u^{*}=\min _{i} u_{i}^{*}$. Note that by decreasing $p_{j}$ by a small amount, one decreases $u_{j}$ below $u_{j}^{*}$ by a small amount, but also increases all other users utilities by a small amount, thus increasing $\min _{i} u_{i}^{*}$. This 
clearly contradicts the fact $\mathbf{p}^{*}$ maximizes the minimum utility. Hence, all $u_{i}^{*}, i=1,2, \ldots, K$ are equal.

Lemmas 3.1 and 3.2 trivially imply that the solution $\mathrm{p}^{*}$ is also unique.

Let us now look at the problem of finding $\mathbf{p}^{*}$. In view of Lemma 3.2, one can rewrite Problem $\mathrm{A}$ as

Problem $\mathbf{A}^{\prime}$ :

Maximize $\kappa$ with respect to $\mathbf{p}$

such that

$$
\frac{\bar{c}}{p_{i}} \exp \left(-\frac{\sigma^{2} \gamma_{t h}}{G_{i i} p_{i}}\right) \prod_{k \neq i} \frac{1}{1+\frac{\gamma+l_{l} G_{i k} p_{k}}{G_{i i} p_{i}}}=\kappa, \forall i
$$

where $\bar{c}=\frac{L R}{M}$.

Next, we introduce a $p_{\min }>0$ such that $p_{i} \geq p_{\min }, \forall i$. This is justified since $p_{i}>0$, however, the choice of $p_{\min }$ depends on the choice of the system parameters. In principle, $p_{\text {min }}$ can be chosen to be as small as possible, as long as $p_{\text {min }}>0$. In practice, $p_{\min }$ will be determined by the device in the mobile transmitter. Also, it is trivial to extend it to the case where each user $i$ has a different $p_{\min }^{i}, i=1,2, \ldots, K$.

After some rearranging of (8), one can now pose the problem as

Problem A1:

Minimize $\delta$

such that

$$
\frac{\sigma^{2} \gamma_{t h}}{G_{i i} p_{i}}+\ln \frac{p_{i}}{p_{\min }}+\sum_{k \neq i} \ln \left(1+\frac{\gamma_{t h} G_{i k} p_{k}}{G_{i i} p_{i}}\right)=\delta, \forall i
$$

Clearly, if the minimum $\delta$ is denoted as $\delta^{*}$, then $u^{*}=$ $\max _{\mathbf{p}} t=\exp \left(\ln \frac{\bar{c}}{p_{\text {min }}}-\delta^{*}\right)$.

This can now be stated as finding a positive vector $\mathbf{p}$ and $\delta$ so that $\delta$ is minimized and $B(\mathbf{p}) \mathbf{p}=\delta \mathbf{p}$ where the entries of the positive matrix $B(\mathrm{p})$ are given by

$$
\begin{aligned}
B_{i i} & =\frac{\sigma^{2} \gamma_{t h}}{G_{i i} p_{i}}+\ln \frac{p_{i}}{p_{\min }} \\
B_{i k} & =\frac{p_{i}}{p_{k}} \ln \left(1+\frac{\gamma_{t h} G_{i k} p_{k}}{G_{i i} p_{i}}\right), k \neq i
\end{aligned}
$$

If one ignores the fact that $B(\mathbf{p})$ depends on $\mathbf{p}$, this can be solved as a Perron-Frobenius eigenvector problem (since $B$ is a positive matrix ensured by the fact that $p_{i} \geq p_{\min }, \forall i$ ).

An iterative algorithm is used to solve this problem starting with an initial vector $\mathbf{p}^{0}=\left(p_{\min } p_{\min } \ldots p_{\min }\right)^{\prime}$ and then iteratively solving for

$$
B\left(\mathbf{p}^{n-1}\right) \mathbf{p}^{n}=\delta^{n} \mathbf{p}^{n}
$$

where $n \geq 1$. This procedure is repeated until $\mathbf{p}^{n}$ does not change. It was seen through computer experiments that this iterative procedure converges to the final solution $\mathrm{p}^{*}$ and the corresponding $\delta^{*}$ quite fast. Similar algorithms are used to solve optimal power control problems in [4] [5]. Later, in Section IV, we provide some numerical results for this algorithm.

\section{B. Sum Power Minimization}

In this subsection, we are interested in a different, but related optimization problem. Minimization of transmitted mobile power is desirable for many reasons including extending battery life, reducing radiation level and reducing cochannel interference in interference limited wireless networks such as CDMA. For voice communications, optimal power control has been studied extensively, often as an optimization problem of sum power minimization with SIR or outage probability constraints [4], [5], [6] (see also references therein). For data communications over wireless, although power control for utility maximization has been studied in [8], we believe that our work is the first to investigate the problem of sum power minimization with outage-based utility constraints for wireless data communication.

We therefore consider the following problem:

\section{Problem B:}

Minimize $\sum_{i=1}^{K} p_{i}$ over $\mathrm{p}$

such that $u_{i} \geq u^{*}, 0<p_{i} \leq p_{\max }, \forall i$, where $u_{i}$ is defined by (7).

Note here again that one can make the minimum utility target $u^{*}$ different for different users in a multi-class service, but here we consider the same target for all users, that is, a single service class. In practice, one can choose the utility targets to be a prescribed fraction of the maximum utility that can be achieved in the system by solving Problem A studied in the previous section. Of course, if some users have their utility targets less than the maximum (minimum). utility achieved by solving Problem A, some other users can have their utility targets higher than this maximum provided the optimization problem is feasible. This is a useful fact for the case when one is interested in a multi-class service.

We now provide a simple intuitive proof of the following Lemma.

Lemma 3.3: Consider Problem B. Suppose it is feasible and there is a feasible power vector $\mathbf{p}^{\dagger}$ such that $u_{i}\left(\mathbf{p}^{\dagger}\right) \geq$ $u^{*}, \forall i$. If $\mathrm{p}^{\dagger}$ is the optimal power vector, in that it minimizes sum power, then $u_{i}\left(\mathbf{p}^{\dagger}\right)=u^{*}$ and $0<p_{i}^{\dagger} \leq p_{m}^{i}$ where $p_{i}=$ $p_{m}^{i}$ is the unique power at which $u_{i}\left(p_{i}, \mathbf{p}_{-i}^{\dagger}\right)$ is maximized, $\mathbf{p}_{-i}^{\dagger}$ denoting the power vector excluding the user $i$, the $j$-th element of the vector being $p_{j}^{\dagger}$.

Proof: Suppose there is a feasible power vector $\mathbf{p}^{s}$ such that all users satisfy their utility targets exactly, that is $u_{i}=u^{*}, \forall i$. Now suppose the $j$-th user is using a power $p_{j}^{s}$ which according to Figure 1 can lie on either side of $p_{m}^{j}$ where $p_{j}=p_{m}^{j}$ is the power maximizing $u_{j}\left(p_{j}, \mathbf{p}_{-j}^{s}\right)$. If $p_{j}^{s}>p_{m}^{j}$, then clearly it can reduce its power to increase its own utility and in the process, increasing other users' utilities as well. Clearly, $p_{j}^{s}$ is not an element of the optimum power vector if $p_{j}^{s}>p_{m}^{j}$. Hence, the $j$-th user can reduce its power until it gets to the power $p_{j}^{l}$ where $p_{j}^{l} \leq p_{m}^{j}$ and $u_{j}\left(p_{j}^{l}, \mathbf{p}_{-j}^{s}\right)=u^{*}$. Notice that reducing $p_{j}$ of course increases other user's utilities beyond $u^{*}$ as well, therefore 
they can also reduce their powers to achieve utility $u^{*}$. This argument can be repeated for any number of users having feasible power solutions which are greater than their corresponding individual utility maximizing power (when other user's powers are kept fixed). Thus the optimal power vector should have all its elements on the left hand side of their individual utility maximizing power values.

Also, at this point, suppose all users are achieving utility targets $u^{*}$. If any user tries to increase his/her utility by increasing his/her power, then that would decrease other users' utilities below $u^{*}$ and therefore they will have to increase their powers again to increase their utilities to $u^{*}$. Clearly, therefore, for the sum power to be minimum, all the target utilities need to be exactly met, that is at the optimal power solution, $u_{i}=u^{*}, \forall i$.

Notice that Lemma 3.3 allows us to solve for the optimal power vector by solving the set of nonlinear equations $u_{i}=$ $u^{*}, \forall i$ with the appropriate projection of the solutions on to the constraint set $0<p_{\min } \leq p_{i} \leq p_{\max }$ with the choice of an appropriate $p_{\min }>0$. The fact that the optimal power vector has all its elements on the left hand side of their individual utility maximizing power (keeping all other remaining users' powers fixed) can also be used during the projection method, depending on whichever happens to be tighter.

One can use any penalty-based optimization methods or even interior point based optimization methods to solve this problem [14]. But here we use a simple iterative technique that solves a nonlinear vector fixed point equation

$$
\mathbf{p}=f(\mathbf{p})
$$

where the individual element $p_{i}=f_{i}(\mathbf{p})$ such that

$$
f_{i}(\mathbf{p})=\frac{1}{\ln \frac{\bar{c}}{p_{i} u^{*}}}\left[\frac{\sigma^{2} \gamma_{t h}}{G_{i i}}+p_{i} \sum_{k \neq i} \ln \left(1+\frac{\gamma_{t h} G_{i k} p_{k}}{G_{i i} p_{i}}\right)\right]
$$

It is easily seen that Brouwer's Fixed Point Theorem [15] can applied here to show that there is a fixed point. However, one cannot prove global contraction to show uniqueness of the fixed point.

We used an iterative procedure $\mathbf{p}^{\mathbf{n}}=f\left(\mathbf{p}^{\mathbf{n}-1}\right), \mathbf{p}^{\mathbf{0}}=$ $p_{\min } \mathbf{1}_{\mathbf{K}}$ with $p_{\min } \leq p_{i}^{n} \leq p_{\max }, \forall i$, where $\mathbf{p}^{\mathbf{k}}$ is the $k$ th iterate of the fixed point equation and $1_{K}$ is a column vector of all ones of dimension $K$. It was seen during our computer experiments that when initialized with small values (such as $p_{\min }$ ), the iterative method (13) always converged to a unique fixed point, while choosing large initial values made some powers diverge away to $p_{\max }$ without the utility constraints being met. Hence our decision to initialize the algorithm with an appropriately chosen small $p_{\min }$.

Noting that there is at least a fixed point, we make the following assumption:
Assumption 3.1: The mapping $\mathbf{p}=f(\mathbf{p})$ given by (13) has a fixed point $\mathbf{p}^{*}$ such that the function $f($.$) is differen-$ tiable at $\mathbf{p}^{*}$ and the Jacobian $f^{\prime}\left(\mathbf{p}^{*}\right)$ has a spectral radius less than 1 .

Remark 1: Note that clearly, without knowing the fixed point, this assumption cannot be verified at the start of the fixed point iterative procedure. However, when the iterations converge (as they do when initialized with small power values), this assumption was successfully verified at the unique fixed point achieved by the iterative algorithm.

It is well known that Assumption 3.1 guarantees local contraction. Therefore we have the following theorem:

Theorem 1: Suppose Problem B is feasible and that Assumption 3.1 holds. Then, the iterative procedure $\mathbf{p}^{\mathbf{n}}=$ $f\left(\mathbf{p}^{\mathbf{n}-1}\right)$ initialized with a $\mathbf{p}^{0}$ close enough to $\mathbf{p}^{*}$ will converge to $\mathrm{p}^{*}$.

Proof: The proof directly follows from Ostrowski's Theorem (see [16], pp. 300-301). An explicit rate of convergence can also be found there.

It was also verified that the use of a MATLAB optimization routine "nlincon" resulted in identical optimal power solutions when initialized with small enough initial conditions.

\section{NUMERICAL STUDIES}

In this section, we provide some numerical results for our solutions to Problem A and Problem B using our iterative methods described in the previous section.

We consider the uplink of a single cell with nine users, similar to that of [8]. User $i$ is at a distance $d_{i}$ from the base station. We choose the distances to be $\mathbf{d}=$ $\left(\begin{array}{lllllllll}250 & 350 & 570 & 620 & 740 & 810 & 880 & 940 & 1000\end{array}\right)$ in metres and $G_{i i}$ is taken to be $\frac{0.097}{d_{i}^{4}}$ as in [8]. We assume that interference is suppressed by a processing gain, $P_{G}$, so that $G_{i j}=G_{j j} / P_{G}$ and $P_{G}$ is varied between 5 and 50 . We also assume $\sigma^{2}=5 \times 10^{-15}$ Watts, $L=64, M=80$ and $R=10 \mathrm{~Kb} / \mathrm{s}$. The minimum frame success rate is taken to be $99.99 \% . p_{\max }$ is taken to be 2 Watts, and wherever necessary (for numerical convenience) $p_{\min }$ is taken to be 0.1 micro Watts.

Figure 2 shows how the maximum achievable minimum utility can be increased with increasing processing gain. The minimum frame success rate for this set of results is chosen to be $99.99 \%$. One can of course increase the minimum required frame success rate and consider higher processing gains to achieve similar utility values. This clearly illustrates the generic value of multiuser detection in conjunction with power control to maximize user utility in wireless data networks with rapidly fading channels.

Figure 3 on the other hand, shows how the total transmitted power required to maintain the target utilities (identical for all users) increases with increasing utility values. The processing gain for this set of results is fixed at 30 and the minimum frame success rate is taken to be $99.99 \%$. 


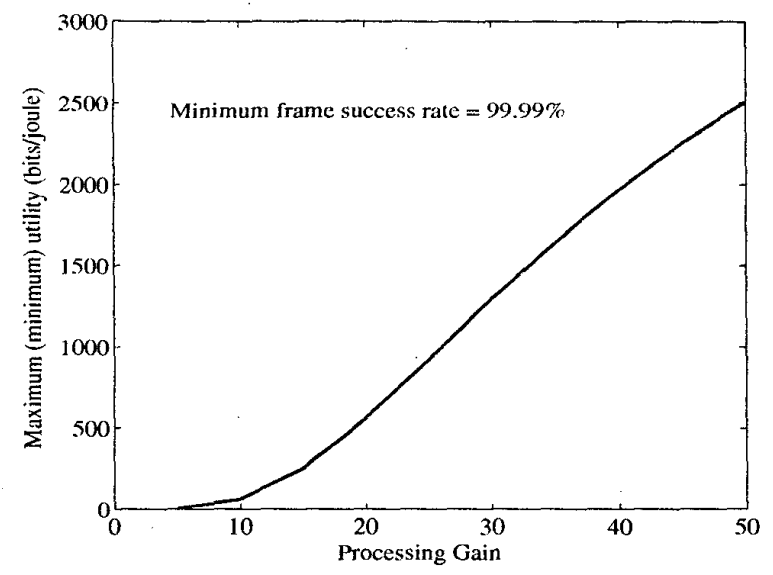

Fig. 2. Maximum Achievable Minimum Utility versus Processing Gain

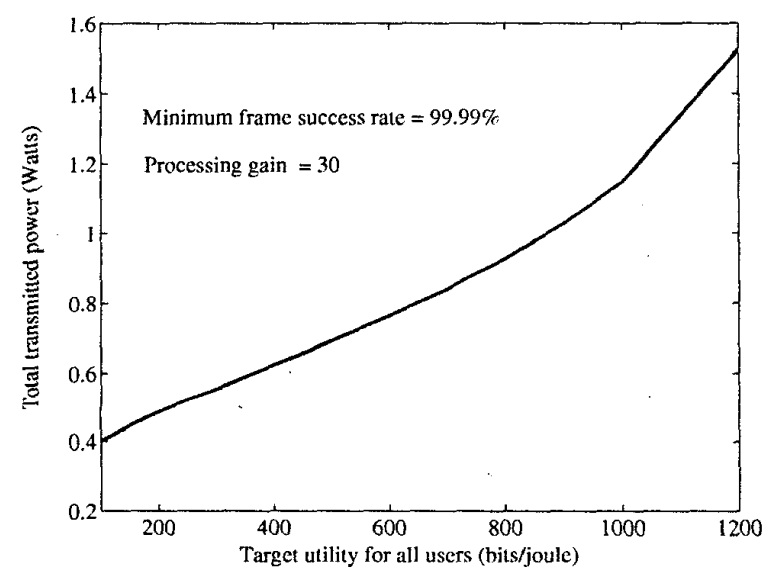

Fig. 3. Total Transmitted Power versus Common Target Utility

\section{Conclusions}

In this paper, we propose an outage-based utility function for transmit power control in an uplink of a single cell of an interference limited wireless data communication system (e.g, CDMA) with Rayleigh fading. We show that one can compute optimal power solutions using simple iterative algorithms that are seen to converge within a few iterations. The results show that one can achieve large utilities for each user by spending a fairly small amount of transmit power if one can employ an appropriate multiuser detector at the receiver to provide a substantial processing gain. Some numerical studies are carried out to study the performance of these algorithms.

\section{REFERENCES}

[1] J. Zander, "Performance of optimal transmitter power control in cellular radio systems," IEEE Transactions on Vehicular Technology, vol. 41, pp. 57-62, February 1992.

[2] R. Yates, "A framework for uplink power control for cellular CDMA systems," IEEE Journal on Selected Areas in Communications, vol. 13, pp. 1341-1347, 1995.

[3] S. Hanly, "An algorithm of combined cell-site selection and power control to maximize cellular spread-spectrum capacity," IEEE Journal on Selected Areas in Communications, vol. 13, pp. 1332-1340, September 1995.

[4] S. Ulukus and R. Yates, "Stochastic power control for cellular radio systems," IEEE Transactions on Communications, vol. 46, pp. 784-798, June 1998.

[5] S. Kandukuri and S. Boyd, "Optimal power control in interference-limited fading wireless channels with outage-probability specifications," IEEE Transactions on Wireless Communications, vol. 1, pp. 46-55, January 2002.

[6] J. Papandriopoulos, J. Evans, and S. Dey, "Iterative power control and multiuser detection with outage probability constraints," in Proceedings of the International Conference on Communications, (Anchorage, Alaska), pp. 2509-2513, May 2003.

[7] D. Goodman and N. Mandayam, "Power control for wireless data," IEEE Personal Communications Magazine, vol. 7, pp. 48-54, April 2000.

[8] C. Saraydar, N. Mandayam, and D. Goodman, "Efficient power control via pricing in wireless data networks," IEEE Transactions on Communications, vol. 50, pp. 291-303, February 2002.

[9] D. Goodman and N. Mandayam, "Network assisted power control for wireless data," MONET, vol. 6, no. 5, pp. 409-415, 2001.

[10] T. Alpcan, T. Basar, R. Srikant, and E. Altman, "CDMA uplink power control as a noncooperative game," Wireless Networks, vol. 8, no. 6, pp. 659-670, 2002.

[11] S. Kim and Y. Lee, "Combined power and rate adaptation in DS/CDMA communications over Nakagami fading channels," IEEE Transactions on Communications, vol. 48, pp. 162-168, January 2000.

[12] G. Stuber, Principles of Mobile Communication. Norwell, MA: Kluwer Academic, 1997.

[13] A. Annamalai, C. Tellambura, and V. Bhargava, "Simple and accurate methods for outage analysis in cellular mobile radio systems - a unified approach," IEEE Transactions on Communications, vol. 49, pp. 303-316, February 2001.

[14] D. Bertsekas, Nonlinear Programming. Belmont, Massachusetts: Athena Scientific, 1999.

[15] E. Zeidler, Applied Functional Analysis: Applications to Mathematical Physics. New York: Springer, 1995.

[16] J. Ortega and W. Rheinboldt, Iterative Solution of Nonlinear Equations in Several Variables. New York: Academic Press, 1970. 